\title{
LA EVALUACIÓN AMBIENTAL ESTRATÉGICA: DESDE EL ENFOQUE DE SU DESARROLLO HISTÓRICO HASTA SU APLICABILIDAD EN LA ACTUALIDAD
}

\author{
D. B. MASSUELA-CALENGA ${ }^{1}$, A. M. OCA-RISCO², M. ULLOA-CARCASSÉS ${ }^{3}$ \\ Escuela Superior Politécnica de Namibe ${ }^{1}$ \\ Universidad de $\mathrm{Moa}^{2}$ \\ amrisco23@gmail.com ${ }^{2}$ \\ Artigo submetido em 12/06/2019 e aceito em 23/12/2019 \\ DOI: $10.15628 /$ holos.2019.8704
}

\section{RESUMEN}

La Evaluación Ambiental Estratégica es una herramienta de la gestión ambiental que se aplica para identificar los impactos ambientales de políticas, planes o programas, así como sus alternativas. El objetivo del artículo fue realizar una investigación documental con el propósito de analizar la evolución histórica desde su aparición hasta nuestros días y profundizar en las diferentes políticas, planes y programas de sostenibilidad ambiental llevadas a cabo a lo largo de la historia, las diferentes normativas y legislaciones vigentes, así como investigaciones y guías metodológicas elaboradas en el mundo referidas a este tema. Se consultaron alrededor de 58 materiales bibliográficos que abordan el tema (libros, artículos, tesis doctorales, tesis de maestría, metodologías, memorias de eventos y leyes) publicados en el periodo comprendido de 1992 a 2019. Se concluye que conceptualmente la EAE precede a la EIA y se constata un aumento progresivo del número y diversidad de enfoques procedimentales y metodológicos. En cuanto avances en aplicación de metodologías y obligatoriedad, se encuentra a la vanguardia el continente europeo, y en el caso del continente africano, particularmente Angola, la EAE es un tema novedoso susceptible de avances en el desarrollo de herramientas de evaluación y control, y su aplicación es limitada por la presencia de obstáculos relacionados con: 1) ausencias de normas y leyes; 2) limitaciones de la capacidad de las instituciones y 3) dificultades relacionadas a la disponibilidad de información. Se propone sea aplicada en este país la metodología creado por la SEAN,debido a las ventajas que presenta.

PALABRAS-CLAVE: Evaluación Ambiental Estratégica, Desarrollo sostenible, Medio ambiente

\section{THE STRATEGIC ENVIRONMENTAL EVALUATION: FROM THE APPROACH OF} HISTORICAL DEVELOPMENT UP TO THEIR APPLICABILITY TODAY

\section{ABSTRACT}

The Strategic Environmental Assessment is a tool of environmental management that is applied to identify the environmental impacts of policies, plans or programs, as well as their alternatives. The objective of the article was to carry out a documentary research with the purpose of analyzing the historical evolution from its appearance until our days and to deepen in the different policies, plans and programs of environmental sustainability carried out throughout history, the different regulations and current legislations, as well as research and methodological guides elaborated in the world referred to this topic. We consulted around 58 bibliographical materials that address the subject (books, articles, doctoral theses, master's theses, methodologies, memories of events and laws) published in the period from 1992 to 2019. It is concluded that the EAE conceptually precedes the EIA and there is a progressive increase in the number and diversity of procedural and methodological approaches. As soon as progress is made in the application of methodologies and obligatory nature, the European continent is at the forefront, and in the case of the African continent, particularly Angola, the SEA is a novel subject that can be advanced in the development of assessment and control tools, and its application is limited by the presence of obstacles related to: 1) absences of norms and laws; 2) limitations of the capacity of the institutions and 3 ) difficulties related to the availability of information. It is proposed that the methodology created by the SEAN be applied in this country, due to the advantages it presents. 


\section{INTRODUCCIÓN}

La preocupación por el medio ambiente y su protección es una cuestión que, desde hace algunas décadas, forma parte de la agenda política, mediática y social de muchos países. Cada vez más, el ser humano es más consciente de su interdependencia para con el medio en el que se desenvuelve, del carácter limitado de los recursos planetarios, de la impronta de los problemas ambientales sobre la vida cotidiana, de la necesidad de conjugar protección ambiental y crecimiento económico apelando al desarrollo sostenible, o de la transversalidad de las cuestiones ambientales. En definitiva, progresivamente, se ha ido tomando conciencia de la necesidad de cambiar el paradigma predominante en la sociedad contemporánea según el cual se asocia evolución y progreso a la mayor capacidad para alterar y dominar la naturaleza, así como con los elevados niveles de consumo de los recursos naturales (Sanz \& Sánchez, 1995).

La Evaluación Ambiental Estratégica, en lo adelante EAE es un instrumento de apoyo a la decisión que se desarrolla en la forma de un proceso, se aplica a decisiones de naturaleza estratégica, normalmente traducidas en políticas, planes y programas, y también constituye un proceso sistemático de identificación, análisis y evaluación previa de impactos de naturaleza estratégica.

Se entiende como impactos de naturaleza estratégica a los que son generados por causas (acciones y decisiones) de naturaleza estratégica y cuyos efectos (consecuencias o resultados de esas acciones) deben ser interpretados bajo una óptica estratégica. Por estrategia, se entiende las principales líneas de acción que permiten alcanzar objetivos de largo plazo, en el marco de principios o supuestos establecidos (Partidario, 2011).

La EAE actúa, preferencialmente, sobre el proceso de concepción y elaboración de políticas, planes y programas y no sobre su resultado y busca influenciar el modo y las prioridades de decisión, así como el ámbito de los vectores considerados relevantes para la decisión. En la EAE las acciones causantes de impacto corresponden a las intenciones de desarrollo, o sea, a la definición del concepto de desarrollo, de un modelo territorial físico pretendido, o de un modelo económico y social con objetivos y metas definidos y, preferencialmente, con una visión de largo plazo y con opciones de desarrollo para el alcance de esos mismos objetivos y metas (Partidario, 2011).

En los primeros años del nuevo mileno, la EAE se ha consolidado como una herramienta imprescindible en los sistemas de gestión ambiental a escala internacional. Del mismo modo, tanto en países desarrollados como en desarrollo, paulatinamente se ha comenzado a incorporar el uso de la EAE como una herramienta formando parte de marcos legislativos nacionales. Sin embargo, la experiencia internacional y la literatura sobre EAE han venido destacando principios de buena práctica en su uso que acentúan la necesidad de adoptar una naturaleza flexible y estratégica, independiente de su escala y ámbito de aplicación (Menéndez, 2017).

Si bien la idea de abordar las preocupaciones ambientales a nivel de proyecto ha tenido una gran aceptación en regiones como el sur de África (Retief, 2008), la implementación de la EAE en este continente ha sido limitada.

Para Angola es de gran importancia el compromiso de consolidar una planeación que integre consideraciones ecosistémicas, sociales, económicas y técnicas en los procesos de formulación y revisión de las políticas, con el fin de que se tomen las mejores decisiones de desarrollo y se asegure 
su productividad y competitividad. Con el desarrollo de nuevos planes, políticas, o programas, que determinan los lineamientos del sector minero, el estado se ha preocupado por incorporar nuevos elementos de análisis como el enfoque ambiental ya que éste permite la participación activa en la formulación y el establecimiento de dichas políticas.

Angola posee grandes depósitos minerales, ubicados en su mayoría en la Provincia de Namibe tales como: La Sierra da Lua; La Sierra da Uimba; La Sierra da Hapa; La Sierra da Picona y la Sierra do Chitovânguael. Los yacimientos cuentan con recursos importantes y de excelente calidad que motivan la aplicación de una metodología para la EAE en la explotación de estos yacimientos, de forma racional y eficiente.

Por lo antes expuesto se realizó una investigación documental cualitativa con el propósito de analizar la evolución histórica desde la aparición de la EAE hasta nuestros días y profundizar en las diferentes políticas, planes y programas de sostenibilidad ambiental llevadas a cabo a lo largo de la historia, las diferentes normativas y legislaciones vigentes, así como investigaciones y guías metodológicas elaboradas en el mundo referidas a este tema. final

Finalmente se describen las investigaciones de la EAE relacionadas con la minería para analizar su evolución y proponer la metodología acertada para Angola teniendo en cuenta las características de este país.

\section{METOdOLOGÍA}

Para la investigación se consultaron un total de 58 materiales bibliográficos, contenidos en libros, memorias de eventos y artículos en revistas científicas; así como tesis doctorales, de maestría, guías metodológicas, que abarcan el periodo desde el año 1992 hasta el 2019.

La revisión bibliográfica se orientó en dos direcciones fundamentales, la información relacionada con el enfoque teórico de la EAE y los trabajos que sobre el tema, desde el punto de vista práctico, se han llevado a cabo.

\section{RESULTADOS Y DISCUSIÓN}

\subsection{Evolución y variabilidad conceptual en la definición de la EAE.}

Desde que a finales de la década de los sesenta del pasado siglo surgió la preocupación por el medio ambiente han aparecido definiciones diversas que han evolucionado hacia el actual enfoque proactivo de la EAE (Gómez, 2007).

Existen diversidad de definiciones sobre las EAE, escritas sobre los principios de participación y transparencia, a continuación se presentan parcialmente algunas de ellas para hacer un análisis de como a través del tiempo se ha perfeccionado este concepto alrededor del mundo.

Transcurridos más de 20 años de existencia, es posible aseverar que la EAE ha tenido una evolución diversa y sujeta a múltiples interpretaciones. Se aprecia una inexistencia de definiciones generalizadas e inequívocas en relación con su propósito, su carácter "estratégico", así como de aspectos procedimentales. No obstante, tanto para comprender esta situación como para precisar su entendimiento, conviene entender su proceso de evolución conceptual y avanzar 
progresivamente hacia una mejor sistematización de sus diversas aproximaciones. A continuación se presenta, ordenadas cronológicamente, las aproximaciones conceptuales que han dominado la discusión científico-técnica durante la última década, ello a partir de las definiciones propuestas por diversos autores a través de publicaciones científicas y documentos de trabajo.

La literatura en EAE ha sido bastante insistente en señalar la ausencia de una definición precisa de la EAE y de sus objetivos, esto ha dado lugar a que en los hechos la EAE asuma una diversidad muy grande de formas, lo que ha llevado a entenderla como una "familia de herramientas en evolución". Esta situación ha dado lugar a que se faciliten diversos esquemas para la clasificación de las EAE realizadas en la práctica, según los objetivos, aproximaciones, y técnicas utilizadas, (Partidario \& Clark, 2000; Dalal-Clayton \& Sadler 2005; Sadler 2008, Bina 2008), o que se faciliten esquemas conceptuales para clasificar las distintas variedades de EAE (Wallington et. al, 2007).

Uno de los primeros conceptos que se tiene sobre EAE es el propuesto por Thérivel et al. (1992) lo cual lo definen como un proceso formalizado, sistemático y comprensivo para evaluar los impactos ambientales de una política, plan o programa y sus alternativas. Incluye la preparación de un reporte escrito de los resultados y uso de las conclusiones en la toma de decisiones.

Otro análisis más técnico y práctico es el recogido por Fevero \& Katz (1996) en su investigación sobre EAE, la describe como "la aplicación de los principios de Evaluación de Impacto Ambiental (EIA) de políticas (ambientales y no ambientales), planes (sectoriales y espaciales) y programas de acción existentes y propuestos". Es decir, los principios y metodologías generales de la EIA son los mismos de la EAE; lo que varía es el ámbito de aplicación de los instrumentos que se van a utilizar, de lo micro (EIA) a lo macro (EAE).

Por su parte, Sadler \& Verhe (1996), plantean que "La EAE es un proceso sistemático para evaluar las consecuencias ambientales de propuestas de iniciativas de políticas, planes o programas para asegurar que ellas se incluyan totalmente y hayan sido tratadas apropiadamente en las fases más tempranas factibles de la toma de decisiones, al mismo nivel que las consideraciones sociales y económicas".

Partidario (1999) expresa que es un proceso sistemático y continuo para evaluar, en las etapas más tempranas de la toma de decisiones, la calidad ambiental y las consecuencias de visiones alternativas e intenciones de desarrollo incorporadas en políticas, planes y programas, asegurando la completa integración de las consideraciones biofísicas, económicas, sociales y políticas relevantes.

Por su parte Brown \& Thérivel (2000) plantean que la EAE es un proceso dirigido a proveer a las autoridades responsables de la formulación de políticas y tomadores de decisión de un entendimiento holístico de las implicancias ambientales y sociales de propuestas políticas, expandiendo el focus mucho más allá de los asuntos que motivaron la formulación de esta nueva política.

Evaluación proactiva de políticas, planes y programas propuestas (o existentes); en el contexto de una visión amplia y un conjunto de metas $u$ objetivos que permitan seleccionar la(s) mejor alternativa(s) para alcanzar un fin deseado, es el concepto que da Noble (2000).

La EAE ofrece una manera práctica y directa de avanzar hacia la consecución de la sostenibilidad Ambiental (acordada en la Asamblea general de la ONU en el 2000). Esta meta 
reconoce la necesidad de la "integración de los principios del desarrollo sostenible en las políticas y los programas de los países". Adicionalmente, la EAE también ayuda a cumplir el plan Johannesburgo de implementación, acordado en la Cumbre Mundial sobre Desarrollo sostenible en 2002, que hizo énfasis en la importancia de "marcos estratégicos y una toma de decisiones equilibrada para hacer avanzar la agenda de desarrollo sostenible"(OCDE, 2006).

Mercier \& Ahmed (2004) plantean la EAE como una aproximación participativa de conducir los asuntos ambientales y sociales para influenciar la planificación ambiental, la toma de decisiones y la implementación de procesos en el ámbito estratégico.

Dalkmann et al. (2004) exponen que en lugar de evaluar los efectos ambientales de una decisión, la EAE debe influir en el proceso de toma de decisiones. Debe estar centrada en las prioridades, asuntos y valores que gobiernan el proceso, y asegurar la integración de ciertos objetivos y valores ambientales dentro de este.

La EAE se consolida como un instrumento de gestión reconocido que, "además de producir información basada en análisis ambientales y socioculturales, constituye una de las principales herramientas de decisión política para abordar tempranamente sus consecuencias, beneficios y riesgos" (IDB, 2004), siendo la última generación de mecanismos de gestión ambiental y planificación económica estratégica basada en una primera generación ya muy desarrollada, las EIA, relacionadas con el crecimiento económico y el desarrollo estratégico de la planeación, la EAE permite analizar el campo político con la mirada ambiental a partir de una multiplicidad de visiones y variables.

Otras organizaciones internacionales también reflexionan sobre este término (Banco Mundial, 2005) "Las EAE, permiten una evaluación comprensiva e integral de los asuntos ambientales, y pueden utilizarse para establecer políticas de desarrollo sectorial o regional ambientalmente idóneas, que de igual forma permitan la viabilidad técnica y financiera de los proyectos del sector".

La Organización OECD-DAC (2006) lo refiere a un proceso definido mediante el cual los tomadores de decisiones consideran los impactos ambientales potenciales durante la formulación, revisión o evaluación de políticas, planes y programas.

Todas las definiciones llevan a considerar incorrecto, o sea, mal concebido y resuelto, cualquier instrumento de planificación: política, plan, programa o proyecto, que no incorpore al medio ambiente, desde el principio, en su concecpción y en su desarrollo, con la misma atención, aunque no con la misma importancia, que los aspectos económicos y sociales (Gómez, 2007).

Más actual es el concepto ofrecido por (Charry, 2010), el cual plantea que la EAE, es un proceso formal que debe ser monitoriado y controlado, sistemático y global para evaluar las posibles repercusiones ambientales de las propuestas políticas, planes y programas durante su proceso de construcción y dado el alto nivel de aplicación debe considerar la integración efectiva de los objetivos ambientales, políticos, económicos y sociales del país, así mismo ser permeada durante todo su proceso por la participación incluyente de la comunidad.

\subsection{Historia y contexto de la EAE.}


La EAE se originó en los países desarrollados alrededor del pensamiento territorial y el desarrollo regional, es decir el uso sustentable de los recursos, a partir de un análisis particular de la oferta de recursos naturales aprovechables en cada espacio especifico, su comienzo se remonta a la Environmental Policy Act (NEPA) de los Estados Unidos de 1969 que exigió la elaboración de informes sobre las consecuencias ambientales de las acciones federales.

En 1981 el Departamento de Desarrollo Urbano y Residencial de EE.UU publicó la guía de evaluación de impacto de áreas extensas (USHUD, 1981) y en 1991 con la Convención sobre Evaluación de Impacto Ambiental de Contextos Transfronterizos y la decisión de la comunidad europea expresa en la directiva 2008/871/CE establecieron los fundamentos para la introducción de la EAE. La directiva Europea 2001/42/CE, ordenó que los miembros de la Unión Europea debieran ratificar el proceso para Julio de 2004.

A continuación se muestran, para cada país elegido (tabla 1), ejemplos significativos de la $E A E$, en cada uno de ellos, donde se integran de manera sistémica los siguientes aspectos:

- PAís: Locación y determinación del área, si es específica o cubre sólo ciertas localidades donde se desarrolla la política y los casos de las EAE.

- ESTATUS LEGAL: Describe la obligatoriedad o no del uso de la herramienta

- ENFOQUE: Descripción del estado y el contenido de la normatividad que se emplea en las EAE

Tabla 1 Situación de la EAE en varios países tomando como base la propuesta de González (2007) tomado de Charry (2010).

\begin{tabular}{|c|c|c|}
\hline PAís & ESTATUS LEGAL & ENFOQUE \\
\hline $\begin{array}{l}\text { ESTADOS } \\
\text { UNIDOS }\end{array}$ & $\begin{array}{l}\text { La EAE en Estados Unidos se exige a } \\
\text { partir de la entrada en regulación de la } \\
\text { Nacional Environmental Policy Act de } \\
\text { 1970: Programatic Environmental } \\
\text { Impact Statement: exigido para todas } \\
\text { las agencias federales que planeen } \\
\text { desarrollar acciones que tengan el } \\
\text { potencial de afectar significativamente } \\
\text { la calidad de vida humana } \\
\text { particularmente, efectos acumulativos } \\
\text { de acciones localizadas } \\
\text { geográficamente en la misma área. }\end{array}$ & $\begin{array}{l}\text { Mediante la } \\
\text { Environmental Quality Act aprobado } \\
\text { en } 1986 \text { se logra un desarrollo } \\
\text { basado específicamente en términos } \\
\text { de EAE, que incluye los siguientes } \\
\text { elementos referidos al enfoque } \\
\text { constitutivo del proceso: Descripción } \\
\text { de la acción, Línea base del medio } \\
\text { ambiente, Evaluación de los } \\
\text { impactos, Alternativas } \\
\text { contempladas e Indicador de } \\
\text { pertenencia. }\end{array}$ \\
\hline $\begin{array}{l}\text { NUEVA } \\
\text { ZELANDA }\end{array}$ & $\begin{array}{l}\text { El Resource Management Act aprobado } \\
\text { en } 1991 \text { obliga a que todas las Políticas, } \\
\text { Planes, y Programas (PPP) se evalúen de } \\
\text { manera que se puedan determinar los } \\
\text { costos y beneficios potenciales para } \\
\text { alcanzar sus objetivos }\end{array}$ & $\begin{array}{l}\text { La EAE se convierte en una } \\
\text { herramienta para alcanzar la } \\
\text { sostenibilidad de planes, políticas y } \\
\text { programas dentro de la puesta en } \\
\text { marcha de los planes nacionales de } \\
\text { desarrollo con el enfoque de la } \\
\text { sustentabilidad. }\end{array}$ \\
\hline
\end{tabular}




\begin{tabular}{|c|c|}
\hline CANADÁ & $\begin{array}{l}\text { La aparición legal e instituida de la } \\
\text { figura de EAE en Canadá surgió desde } \\
\text { finales de los } 80 \text { s obedeciendo a la } \\
\text { aplicación de principios de evaluación } \\
\text { de impacto ambiental a los PPP, en } 1999 \\
\text { se redactó la "Cabinet Directive on SEA" } \\
\text { que especifica que los Ministerios } \\
\text { deben desarrollar procesos de EAE para } \\
\text { propuestas de PPP, cuando la propuesta } \\
\text { requiera aprobación de un ministerio. }\end{array}$ \\
\hline
\end{tabular}

REINO UNIDO Documentos Guía expedidos por los ministerios competentes al desarrollo de propuestas que involucren directamente variables ambientales

\begin{abstract}
La concepción del proceso metodológico de EAE en Canadá aplica de fondo el "Principio de Precaución" y maneja la teoría evolutiva de los estudios ambientales consignándolos principios de evaluación de impacto ambiental a nivel de PPP.
\end{abstract}

Se desarrolla un documento, el "Environmental appraisals" que define el enfoque general que deben tener las políticas del gobierno central y sumado a esto acondiciona los planes de desarrollo de las autoridades locales (IAvH, 2007).
COMUNIDAD Se define dentro de la normativa EUROPEA expedida por la directiva 2001/42/CE del Parlamento Europeo y del Consejo de 27 de junio de 2001 relativa a la evaluación de los efectos de determinados planes y programas en el medio ambiente.
Se puntualiza como un Tratado de la Comunidad Europea y obliga a sus instituciones, licitantes y contratantes a integrar requerimientos en el enfoque de la protección ambiental en la definición e implementación de sus políticas y actividades, para promover el desarrollo sostenible

Los actuales enfoques regionales de las EAE tienen un camino conservacionista y se aplican principalmente a efectos de la biodiversidad caso IAvH con las guías de incorporación de biodiversidad en las políticas (Toro, 2009), o como refiere el caso de Costa Rica, donde la herramienta fue útil en la definición de la política de BioTurismo.

La mayoría de los estudios se han encaminado hacia los procesos de conservación y biodiversidad, con un enfoque alejado de las decisiones 
políticas orientadas a procesos

económicos.

ÁFRICA DEL Entre los ejemplos más notorios está SUBSAHARA Sudáfrica que ha desarrollado guías para realizar EAE con una visión descontextualizada de la herramienta ya que su orientación es identificar oportunidades y restricciones que el medio ambiente impone para el desarrollo, siendo uno de los objetivos centrales de la EAE enfocarse en los impactos del desarrollo sobre el medio ambiente.
El alcance de la EAE se define en el contexto amplio de procesos ambientales, integrando consideraciones biofísicas, sociales y económicas en la formulación, con orientación al desarrollo sostenible.

\subsubsection{EAE en el contexto internacional}

La revisión sobre los principales avances en la EAE en el contexto internacional, se fundamentan en aspectos importantes que puedan aclarar y confrontar el panorama nacional con otras experiencias exitosas a nivel global.

\section{Europa}

En el caso del continente Europeo, la Directiva Europea 2001/42/EC para la Evaluación Ambiental Estratégica es aplicada para Planes públicos y Programas. El principio es asegurar que éstos tengan efectos significativos en el ambiente, antes de la aprobación de los mismos. La consulta pública es considerada la característica fundamental de los procesos de evaluación ambiental (European Comission Environment, 2014).

Finlandia se destacó con cerca de 1500 procedimientos de EAE anualmente realizados, el Reino Unido tenía entre 400 y 500 en proceso en 2008 y Francia realizó cerca de 400 en 2007, solamente referente a los planes de uso de la tierra (Oberling, 2013).

Gran Bretaña se destaca por su fuerte sistema de planificación, teniendo la EAE (llamada Evaluación de Impacto Sustentable) aplicada para políticas y planes (MMA, 2002).

Irlanda pasó a exigir a EAE desde 2001 en una directiva y en 2004 fue transformada en ley, para programas y planes que tienen impactos socio-ambientales significativos, incluyendo la generación de energía y actualmente cerca de 300 EAE empezaron a ser desarrolladas en este país con un $80 \%$ relacionado con el uso del suelo, $7 \%$ sobre el agua, $6 \%$ sobre la energía y $4 \%$ sobre la pesca (EPA, 2012).

\section{América}

Otros países vienen desarrollando la EAE voluntariamente, como Canadá, que posee un decreto que refuerza el papel de la EAE en la toma de decisiones de los PPP de efectos ambientales importantes, de departamentos y agencias federales, a través de la Agencia de Evaluación Ambiental Canadiense. Esa agencia realiza las Evaluaciones de Impactos considerados significativos 
por el gobierno federal, considerando la acumulación de los impactos de manera participativa, integrada y transparente (de acuerdo con el Canadian Environment Assessment Act) (CEAA, 2014).

En América Latina la EAE aún está se estableciendo, incentivada por organizaciones multilaterales de desarrollo económico y social tales como el Banco Mundial, la Comisión Económica para América Latina y Caribe (CEPAL), Comisión Centroamericana de Medio Ambiente y Desarrollo (CCAD) y el Programa de las Naciones Unidas para el Medio Ambiente (PNUMA), (Espinoza, 2011).

Ya la iniciativa para la Integración de la Infraestructura Regional Sur Americana (IIRSA), una organización multinacional, multisectorial y multidisciplinar que desempeña el papel de coordinar 12 gobiernos de América del Sur, bien como las instituciones financieras multilaterales: Banco Interamericano de Desarrollo (BID), Comisión Andina de Fomento (CAF) y Organización para la Cooperación y Desarrollo Económico (OCDE) junto con el sector privado, desarrolla un programa en conjunto que busca promover la integración de la infraestructura de transporte, energía, turismo y telecomunicaciones de los países de América del Sur y desarrolla la EAE entre ellos, como un complemento de la EIA de proyectos financiados por estas instituciones. El Centro de Estudios del Desarrollo (CED), con sede en Chile, también auxilia a los países latinoamericanos con la capacitación para la adopción de la EAE, entre otras iniciativas dirigidas a fomentar el Desarrollo Sostenible (CED, 2010).

Del total de 20 países latinoamericanos, 7 poseen una reglamentación formal que integra la EAE, referente al análisis ambiental de Políticas, Planes y Programas (PPP) para la organización territorial como en Uruguay, con ley 18.308/2008 (CED, 2010), Costa Rica Decreto Ejecutivo $32.967 / 2006$ (Bayo, 2009) y Chile - Ley 20.417/2010 (CED, 2010), siendo estos últimos enfocados al Ordenamiento Territorial (OT).

Brasil, Guatemala y Venezuela poseen acuerdos gubernamentales o proyectos de ley para uso de la EAE. República Dominicana y El Salvador (Decreto 17/1998) incentivan la aplicación de la EAE para el análisis de los PPP en la administración pública y Cuba también prevé la EAE para PPP dentro de su política ambiental (CED, 2010).

Los países latinoamericanos vienen avanzando en el proceso de adopción y reglamentación de la EAE aunque todavía de manera pausada, se han logrado algunas experiencias exitosas como el Plan de Reforma del Saneamiento de Colombia y de la Argentina, estimulados por el Banco Mundial (Enriquez \& Sanchez-Triana, 2005), entre otras iniciativas (Sinclair et al. 2009).

Asia

La EAE también ha sido utilizada en Asia, donde hay una creciente demanda por energía, cuya proyección para 2030 es de ser el doble del consumo actual, incluyendo la región del pacífico, de acuerdo con el Banco Asiático de Desarrollo (ADB, 2014). Así, los gobiernos de los diversos países empiezan a tener que pensar en la planificación estratégica para tal demanda.

De los once países en la región de Hong Kong (Special Administrative Region - SAR), China, Vietnam, Corea y Japón están más avanzados con relación a la EAE, aplicándola desde los años 90. Esa región fue la primera en desarrollar un mandato legal para algunos tipos de $E A E$, a través de una directiva para las políticas y estrategias. Actualmente es obligatorio que una EAE sea anexada a un documento de política o plan a ser presentado para aprobación del Consejo Legislativo de finanzas y al Consejo Ejecutivo de políticas (MRC, 2010). 
China es uno de los pocos países asiáticos que han adoptado oficialmente la EAE (Tao et al. 2007). El requisito legal de aplicar la EAE a los planes y programas en China se estableció en 2003 tras la implementación de la Ley de Evaluación de Impacto Ambiental. La EAE en China a menudo se conoce como Evaluación de Impacto Ambiental del Plan (PEIA), o plan EIA; los términos se usan indistintamente (Bina et al. 2011; Wu et al. 2011).

Corea del Sur, China y Japón, por ejemplo, tiene una base bien establecida y buen registro de aplicaciones de EIA y EAE a nivel de proyecto, programa y plan,

Como en muchas otras regiones del mundo, la EAE es un concepto nuevo en la región de Asia oriental y el Pacífico. A partir de 2005, solo China, Japón, Corea y Vietnam tenían acuerdos legales y requisitos en cierta medida, para la aplicación de la EAE a nivel nacional o local (Banco Mundial 2005), pero este hecho probablemente ha cambiado ya que cada vez más países han comenzado a intergar la práctica de EAE en diferentes niveles.

La aplicación de la EAE antes del inicio del proyecto ha sido importante en el contexto de la India, ya que los resultados de estas evaluaciones han demostrado los beneficios de entregar la información necesaria para facilitar la toma de decisiones y reducir la necesidad de EIA. La EAE en este país también ha demostrado ser útil a nivel de plan y programa al proporcionar una vista de las cuestiones ambientales y sociales para una evaluación amplia de los impactos acumulativos de proyectos propuestos, antes de su implementación en alguna área protegida del país. (Rajvanshi \& Mathur 2005).

Así, la EAE ha sido aplicada en diversos países del continente asiático, sobre todo aplicados a PPP tales como el Plan de la carretera Hubei, China, el plan detallado del área de Daka, Bangladesh y el National Trade Corridor Improvement Program (NTCIP) de Pakistán (Dalal-Clayton \& Sadler, 2005).

\section{Oceanía}

La Mancomunidad de Australia, Victoria, Australia Occidental y Nueva Zelanda poseen algunas disposiciones legales de carácter permisivo relativas a la EAE dentro de los requisitos de la EIA. A pesar de esto, y un reciente crecimiento en el interés en tales disposiciones, muy pocos informes de EAE se han presentado hasta la fecha. Victoria (en una ocasión), Nueva Zelanda (en una ocasión) y Australia Occidental (en dos o tres ocasiones) han requerido y evaluado proyectos. Wood (1992)

Los requisitos formales para la evaluación ambiental estratégica en Australia y Nueva Zelanda datan de 1974, en ambos paises dicha estrategia se deriva de la Ley de Política Ambiental Nacional de Estados Unidos 1969 (NEPA), dada que esta ley contiene ideas esenciales adpatables a la EAE.

Los paises de Oceanía han mostrado especial interés en la aplicación de la EAE, ejemplo de ello es la participación de Australia, Nueva Zelanda y Canadá en la cumbre tripartista sobre la evaluación de políticas ambientales en Wellington en 1991, donde es creado el Consejo de Conservación Ambiental Australia-Nueva Zelanda (ANZECC). Este Consejo define los requerimientos legales para la aplicación de la EAE en la Mancomunidad de Australia, Victoria, Australia Occidental y Nueva Zelanda, Wood (1992).

África 
El medio ambiente de África ha sido deteriorado constantemente, con la pobreza como uno de los principales síntomas de esa degradación. Altos niveles de pobreza en combinación con casos de variabilidad climática y desastres naturales, así como debilidades internas institucionales han hecho a los africanos más vulnerables físico, psicológico y económicamente. Pobre desempeño económico y débil institucionalidad y marcos jurídicos, así como la sobreexplotación de los recursos naturales y humanos contribuyen al aumento de la vulnerabilidad (Banco mundial, 2005).

El contexto para la Evaluación Ambiental Estratégica (EAE) en África se ha establecido mediante una serie de iniciativas estratégicas que se han desarrollado a nivel continental e internacional. Es importante destacar que la Nueva Alianza para el Desarrollo de África (NEPAD) ha instituido una visión para el crecimiento liderado por África que apunta a promover la renovación del continente y abordar los problemas actuales.

Si bien la idea de abordar las preocupaciones ambientales a nivel de proyecto ha tenido una gran aceptación en regiones como el sur de África (Retief, 2008), la implementación de la EAE en África ha sido limitada. Sin embargo, se están desarrollando cada vez más marcos políticos y legales para la EAE o para procesos de planificación estratégica que incorporan ciertos elementos de la EAE. Por ejemplo, se hace referencia a la evaluación de políticas y programas en la legislación ambiental en países como Benin, Burkina Faso, Madagascar, Djibouti, Senegal y Mozambique (Dalal-Clayton \& Sadler, 2004). Además, hay varios ejemplos de procesos de EAE que se han llevado a cabo o que están actualmente en curso. Estos incluyen una EAE del Proyecto de Azúcar Caprivi en Namibia, la EAE para el Puerto de Ciudad del Cabo en Sudáfrica y la EAE de la Estrategia de Reducción de la Pobreza de Ghana (Dalal-Clayton y Sadler, 2004). Las pautas para la EAE también se han producido, por ejemplo, las publicadas por el Banco Africano de Desarrollo en 2003 (denominadas Directrices de Evaluación de Impacto Estratégico) y las publicadas por el Departamento de Asuntos Ambientales y Turismo (DEAT) en Sudáfrica en 2000.

El número de países africanos que han aprobado disposiciones para la EAE también está en aumento, por ejemplo, la legislación promulgada por Botswana en 2001 y Tanzania en 2004 (DalalClayton \& Sadler, 2005).

En el análisis de la literatura consultada no se encontró la existencia de una legislación para la EAE en Angola.

\subsection{Investigaciones relacionadas con la $\mathrm{EAE}$}

En los últimos años se han realizado innumerables investigaciones a nivel internacional acerca de la EAE y el papel que desempeñan en la protección del medio ambiente.

(Clark 1997) analiza la evolución que ha seguido en distintos países la EAE y los principios básicos que la sustentan y la componen, tales como "screening", "scoping", información básica, predicción de impactos, evaluación de impactos, informe, revisión y supervisión. Asimismo, examina las diferencias de procedimiento entre EAE y EIA, destacando temas tales como la confidencialidad, constitucionalidad, deficiencias de procedimiento, relación proponente-autoridad, restricción de competencias y otras y describe distintos modelos para la EAE. Finalmente, explora la aplicación de la EAE a diferentes escalas tales como la evaluación de políticas, planificación territorial y gestión 
de recursos, evaluaciones ambientales a nivel regional, evaluación ambiental acumulativa, sectorial, de privatizaciones, de ajustes estructurales, de presupuestos nacionales, de temas globales y tratados.

Nigel et al. 2000 describen la base conceptual y el enfoque de la EAE tal como se ha desarrollado en Sudáfrica. Dicho enfoque se centra en el efecto del medio ambiente en el desarrollo, en lugar del impacto del desarrollo en el medio ambiente. Se analizaron estudios de casos relacionados con la EAE en Sudáfrica y su contexto. Se expone que aunque Sudáfrica no tiene una legislación referida a la EAE, sin embargo, con el fin de proporcionar un enfoque más eficiente a esta estructura, se han redactado directrices para su aplicación a nivel de planificación y programa. Los autores plantean que los tres conceptos clave que sustentan la EAE en Sudáfrica son; especificidad para cada contexto analizado, integralidad y sostenibilidad, estos conceptos están respaldados por uel conjunto de principios de la EAE.

Por su parte, Charry (2010), realizó su investigación en el marco de los Estudios Ambientales enfocándose en el estado y desarrollo de la segunda generación de estos llamada Evaluación Ambiental Estratégica (EAE), tomando como referencia los avances que ha realizado en Colombia en la materia y principalmente los ejercicios del subsector petrolero, dirigiendo sus esfuerzos investigativos a una revisión de los procesos que actualmente lleva el país referente a los estudios de impacto ambiental y los cambios producidos a través del tiempo, haciendo énfasis en las herramientas de aplicación de estos y su perspectiva de transformación. Como resultado hizo un análisis de la situación actual del país frente a los estudios ambientales de segunda generación (EAE), sus aplicaciones más recientes, desarrollando una propuesta de metodología de aplicación de EAE que incluye modificaciones acordes con el contexto colombiano, para el caso del estudio que compete, convirtiéndose en una herramienta importante de manera que pueda ser modificada para otros subsectores.

Mientras tanto Partidario (2011) presenta el concepto de Evaluación Ambiental Estratégica (EAE) como un instrumento facilitador de decisiones de naturaleza estratégica, cuyo objetivo es asegurar la integración de las cuestiones ambientales en un contexto de sustentabilidad, con amplio alcance y de largo plazo. Se hace referencia a su evolución, a las múltiples perspectivas que se han desarrollado sobre EAE y la influencia de las diferentes escuelas de planeación; se presentan, además, cuatro modelos explicativos de los diferentes abordajes del EAE y se justifica una perspectiva de EAE como un abordaje metodológico basado en una lógica de pensamiento estratégico y ajustable a diferentes procesos de decisión, derivado de un modelo de EAE centrado en la decisión. Se destacan las ventajas de este enfoque por medio de la comparación a las formas más tradicionales de las metodologías que siguen la lógica de la EIA.

(Ahumada et al. 2010) abogan por la adopción de la EAE como mecanismo práctico para hacer más operativa la sustentabilidad ambiental. Aborda la situación actual del proceso de planeación del desarrollo y la EIA como línea de base para realizar la propuesta de implementación de la EAE en México. Finalmente, proponem la reforma a la Ley General del Equilibrio Ecológico y la Protección al Ambiente para adicionar la EAE como un instrumento de la política ambiental de carácter preventivo que evalúe las repercusiones ambientales de ciertos programas sectoriales, institucionales, regionales y especiales considerados en la Ley de Planeación. 
En su artículo "Evaluación ambiental estratégica: Instrumento para la planificación territorial urbana" los autores tienen por finalidad relevar la EAE como una herramienta útil para la generación de los Instrumentos de Planificación Territorial en la Región de La Araucanía. Se identificaron y caracterizaron los distintos niveles de planificación urbana, según lo establecido por la Ley General de Urbanismo y Construcciones; contrastando el ingreso de estos al Sistema de Evaluación de Impacto Ambiental (SEIA) definido en la Ley 19.300 de Bases del Medio Ambiente y su posterior modificación a través de la Ley 20.417. Para contrastar ambos procedimientos se desarrolló un proceso exploratorio descriptivo, utilizando tanto fuentes primarias, a través de la aplicación de entrevistas a actores claves como secundarios. (Molina \& Escalona, 2012)

Marrero et al. 2013, plantean que la aplicación de la EAE en Cuba es reciente y cobra importancia entre otras cosas, por su vinculación con el ordenamiento ambiental y el desarrollo sostenible de los territorios. El objetivo del artículo fue establecer un marco conceptual sobre el tema así como proponer los procedimientos metodológicos para llevar a cabo ese tipo de estudio en el país. La propuesta de la metodología de elaboración de la EAE se sustenta en la experiencia nacional e internacional más conocida, y se tuvo en cuenta las condiciones de Cuba, de los conceptos y principios que aplican. Finalmente se propone que la Autoridad Ambiental de Cuba evalúe la propuesta de Guía Metodológica para la ejecución de EAE que se presenta en dicho trabajo, para su aplicación temporal en el país, además conciliar los resultados de la EAE a los ordenamientos territoriales y ambientales de los territorios donde se realicen, con el objetivo de actualizar y mejorarlos sistemáticamente a partir de las nuevas Políticas, Planes y Programas.

Lobos \& Paridario (2014) apuntaron a confirmar y justificar una brecha existente entre el desarrollo teórico de la EAE y su práctica. Plantean que aun no es evidente cómo y cuándo la práctica de SEA evolucionará en la misma dirección de la teoría, pero los informes revisados muestran que todavía existe evidencia significante de resistencia al cambio. La principal preocupación de los autores en su investigación es hasta qué punto esta tendencia puede afectar la credibilidad de la EAE en relación con su capacidad para mejorar la forma en que se toman las decisiones de política y planificación. Los investigadores consideran que las razones de esta brecha entre teoría y práctica pueden ser muchas, pero concentraron su argumento en torno a dos razones principales: la resistencia al cambio de los practicantes y desafíos creados por sistemas complejos inevitables.

Pérez (2017) en su investigación se centra en el estudio de la relación entre la importancia de la protección ambiental y la evaluación anterior de aquellos planes y programas con incidencia sobre el medio. Por ello, se focaliza la atención en la evaluación ambiental estratégica como un instrumento cuyo procedimiento se encarga de la aprobación de dichos planes y programas, formando una unidad imprescindible. Especialmente, se trata de enfatizar en su actual situación en el Estado Autonómico, en general, y en la Comunitat Valenciana, de España.

\subsubsection{Guías de evaluación ambiental estratégica}

En otro orden de investigación, se han desarrollado guías a nivel mundial sobre EAE en varias regiones o países, el objetivo de estas guías es difundir, facilitar y viabilizar el uso de las EAE por parte de los promotores de las iniciativas, suministrando la información que pueden requerir las autoridades, los políticos, los planificadores y los posibles interesados del proceso.

En la guía de EAE de Jiliberto \& Bonilla (2009), el propósito fundamental fue facilitar una estructura de apoyo para desarrollar una evaluación ambiental estratégica de planes y programas 
que mejorara la integración de la dimensión ambiental y de sostenibilidad en planes y programas. El documento se estructura en tres cuerpos principales además de una introducción, un capítulo de anexos y un glosario, que en conjunto ofrecen una referencia completa para el planificador y el evaluador ambiental. En ella se presentan un conjunto de herramientas específicamente diseñadas para apoyar la consecución de las actividades que suponen la aplicación de la EAE.

En 2015 el Ministerio del Medio Ambiente Oficina de Evaluación Ambiental de Chile aprobó la Guía de orientación para el uso de la $E A E$, con el objetivo de disponer de una herramienta de apoyo con orientaciones conceptuales y metodológicas para la aplicación en procesos de formulación de políticas y planes de carácter normativo e instrumentos de ordenamiento territorial, en el marco de la normativa vigente en Chile. Dicha guía se basa en el enfoque de pensamiento estratégico (promovido por Partidario, 2007), buscando la integración de criterios de desarrollo sustentable y objetivos ambientales en los procesos de formulación de políticas y planes.

En 2012, Partidario elaboró la guía de Mejores Prácticas para la Evaluación Ambiental Estratégica: Orientaciones metodológicas para un pensamiento estratégico en EAE, cuyo objetivo consistió en ofrecer orientaciones prácticas sobre cómo usarla de forma innovadora y orientada a la sustentabilidad siguiendo el pensamiento estratégico. Las orientaciones se aplicaron a todas las acciones que son determinadas por objetivos estratégicos de largo plazo. El enfoque se basó en un modelo de pensamiento estratégico de naturaleza integradora, holística y transversal (multasectorial e interdisciplinaria) que se ilustró con ejemplos de casos tomados de Portugal y otros países. Esta guía es una versión modificada y actualizada de la pauta metodológica adoptada y publicada en 2007 por la Agencia Portuguesa de Ambiente en la cual se aclaran conceptos, se explica la ejecución real de una EAE como evaluación de carácter estratégico, se presentan las técnicas más utilizadas y se exponen ejemplos de cómo puede ser al mismo tiempo un instrumento de carácter estratégico para asegurar el cumplimiento de la legislación europea y portuguesa.

La Organización para la Cooperación y el Desarrollo Económicos (OCDE) en 2006 creó una guía de buenas prácticas en la cooperación para el desarrollo. Esta guía ofrece un marco flexible alrededor de unos principios acordados comúnmente, para ayudar a impartirle coherencia a la práctica de la EAE. Dentro de los objetivos de la guía se persigue: Describir la importancia de considerar el medioambiente para alcanzar el desarrollo económico sostenido y la reducción de pobreza, explicar la contribución del medioambiente a la sostenibilidad de una serie de intervenciones en el desarrollo y describir el consenso emergente sobre el valor de la EAE en la cooperación para el desarrollo, aprovechando la experiencia internacional, que está en evolución, incluyendo los principios y procedimientos sobre cuya importancia existe un acuerdo general.

Otra de las Organizaciones que elaboró una guía para la EAE fue el Banco Interamericano de Desarrollo, (Kulsum \& Sánchez-Triana, 2009) que elaboró la guía para la aplicación de la EAE como una herramienta de gestión, la cual constituye una herramienta conceptual con un formato común, simple y práctico de apoyo a la aplicación de la EAE en el BID. La Guía aporta criterios importantes para el análisis de viabilidad ambiental y sociocultural de las decisiones a nivel de políticas, planes y programas (PPP), la identificación, evaluación y revisión de riesgos y oportunidades ambientales y socioculturales de carácter significativo, elaboración de estrategias y planes de acción de riesgos y oportunidades detectadas y la definición de capacidades institucionales para llevar adelante tanto una aplicación de EAE como la gestión de sus resultados. 
En América Latina la Oficina Regional para Mesoamérica. La Unidad de política y gestión ambiental, UICN/ORMA, (2007) elaboró los Lineamientos para la aplicación de la Evaluación Ambiental Estratégica en Centroamérica (UICN). Esta publicación constituye una primera propuesta para la aplicación, de manera gradual, de la EAE en los países centroamericanos, la cual busca un punto de partida para la discusión y el perfeccionamiento de una herramienta de trabajo de gestión ambiental, además sirve de orientación y referencia en la labor de integrar la variable ambiental y social en los PPP. Cuenta con tres capítulos, en el primero se expone el proyecto de Evaluación de Impacto Ambiental en Centroamérica, la importancia y las principales definiciones de los términos básicos utilizados; en el segundo, se exponen criterios de la Evaluación Ambiental Estratégica como un instrumento de gestión preventiva y en el tercero, se ilustran los lineamientos generales para la aplicación de la EAE en Centroamérica, así como el procedimiento metodológico sugerido para llevar a cabo la integración ambiental en la planificación de PPP.

En la guía metodología de la Unión Europea varios de los 25 países que la conforman ya han internalizado completamente la Directiva 42/2001 en su legislación. Dos países Alemania e Italia tienen leyes nacionales sobre EAE pero al ser estados federales y regionales respectivamente aún deben internalizar a esos niveles la Directiva Europea 42/2001. Austria es otro país donde se ha integrado parcialmente dicha Directiva. La UE ha sido lo suficientemente flexible como para permitir que las leyes nacionales tengan características propias. En algunos casos como España, Irlanda, Portugal, Eslovenia y Austria, la Ley internalizada permite a la EAE influenciar en la preparación de las PPP desde el momento de su conceptualización por lo que estaríamos ante una metodología centrada en la decisión estratégica. La Directriz 42/2001 propone siete pasos para la realización de la EAE Jiricka (2008).

La EAE propuesta por el Banco Mundial, está basada desde sus inicios también en directivas operacionales de EIA. Sin embargo ha evolucionado con el tiempo utilizando una gama de diferentes tipos de evaluaciones ambientales como un intento de mirar a las actividades sectoriales en lugar de proyectos individuales y centrar sus resultados en la mejora del marco de gobernabilidad. Las evaluaciones ambientales sectoriales, sin embargo, tienen un carácter mucho más estratégico y se consideran adecuados para su uso en el diseño de programas de inversión sectorial. La experiencia del Banco Mundial en lo que respecta a la aplicación regional y sectorial de evaluaciones ambientales es bastante amplia, en particular en lo que se refiere a evaluaciones ambientales sectoriales.

La intención ha sido incluir evaluaciones ambientales, como parte de la rutina de los estudios sectoriales, proporcionando a los planificadores de la estrategia más sólida ambiental y de sostenibilidad para alcanzar los objetivos de desarrollo de la estrategia. Dentro de la experiencia del Banco Mundial, las operaciones de privatización y ajuste estructural pasaron por el proceso de EAE. Considerando que su enfoque hacia el análisis de impactos y en base a ello plantea mejoras institucionales para su manejo, esta metodología estaría más vinculada al Modelo de EAE basado en impactos - Paralelo (Facetti, 2003).

La metodología propuesta por el SEAN, a su vez, considera según sus creadores como un enfoque práctico para la realización de la EAE. Esta plataforma fue desarrollada en 1999 para la Organización Holandesa para la Cooperación para proyectos de asistencia enfocados en la reducción de la pobreza, con financiamiento del Ministerio de Relaciones Exteriores de Holanda y se la utiliza desde 2002 como un apoyo, capacitación y servicios de consultoría a varios países, como 
Guatemala, Nicaragua, Ghana, Cabo Verde, Honduras, Zimbabwe, Camerún y Croacia. Su metodología consta de 10 pasos, con énfasis en el proceso participativo. Sus medidas son: la preparación, scoping, el trabajo de campo, la síntesis y la vigilancia. Esta metodología al dar un énfasis a la determinación de impactos, también tendría una aproximación al modelo de EAE basado en EIA-Paralelo (Facetti, 2003).

Los países miembros de la OCDE adoptaron una Declaración en París estableciendo un plan para mejorar la forma de la cooperación para el desarrollo, por lo que crearon la metodología de la OEC, donde el objeto de la misma es ser más eficaz. La Declaración de París también compromete a los donantes y los países socios a "desarrollar y aplicar los enfoques comunes para la evaluación ambiental estratégica". Esta Metodología permite influenciar limitadamente en la preparación de las PPP, dando una fuerte influencia al proceso de participación y de buen gobierno. Nos encontramos ante una metodología EIA-Paralela (Facetti, 2003).

\subsubsection{EAE en minería}

El proceso de evaluación estratégica en la actividad minera reside en combinar y aplicar los conceptos de actuaciones, recuperación y sostenibilidad dentro de un marco estratégico de desarrollo de dicha actividad, para conseguir los objetivos iniciales propuestos en el proyecto.

A pesar de que se han realizado estudios sobre EAE en minería, aun es insuficiente su grado de impletanción en esta actividad económica.

Algunos de los ejemplos donde se aplica la EAE en activiades mineras, es el caso de:

Pineda (2006) el cual aplica la metodología de la EAE, al plan de exploración y explotación de hidrocarburos costa afuera en el Mar Caribe, así como identifica los principales criterios ambientales que se deben tener en cuenta al momento de ejecutar dicho plan. Realiza un análisis de las principales actividades que comprenden el plan de exploración y xplotación, así como una revisión bibliográfica para identificar los aspectos ambientales relevantes en los cuales se hace necesario incluir criterios ambientales que contribuyan al mejoramiento del proceso de toma de decisiones, garantizando un equilibrio entre los aspectos económicos, sociales y ambientales que asegure la ejecución adecuada del plan y a su vez sirva de base para la formulación de futuros procesos de planificación en los cuales el componente ambiental este introducido desde etapas tempranas de la planificación.

En su articulo "Necesidad de una EAE de la minería metalífera en Argentina" (Pirillo et al., 2012) plantean que existen exigencias de aplicación de procedimientos de EIA, generalmente utilizados para obtener el permiso de construcción y operación de los proyectos mineros en Argentina. Sin embargo, no existe hasta el momento, legislación, regulaciones o guías de procedimientos para la realización de Evaluaciones Ambientales Estratégicas (EAE) en dicho país. Los autores sugieren la elaboración de una EAE para la minería a nivel nacional, de modo que se pueda evaluar la sostenibilidad de la actividad, analizando alternativas de explotación, impactos acumulativos, procedimientos administrativos, participación ciudadana, desarrollos regionales, prevención de conflictos, entre otros aspectos de vital importancia para el desarrollo de esta actividad. En este trabajo, se examinan brevemente, algunos requerimientos legales ambientales, impactos sobre los recursos naturales, transferencia a las comunidades aledañas, algunos conflictos 
asociados y se ponen en evidencia los beneficios que aportaría esta herramienta de evaluación para avanzar hacia una gestión sostenible de los recursos minerales.

Por su parte, Calzada (2014) en su tesis doctoral propone una metodología adecuada para la evaluación de los impactos generados por los proyectos mineros buscando su integración al entorno paisajístico; para realizar un adecuado estudio del paisaje, y del efecto paisajístico, de tal forma que el resultado de la introducción del proyecto en el entorno ambiental sea mínimo, o con un impacto moderado y compatible con el entorno. En la investigación, además de establecer una metodología que permite la valoración objetiva y realista de los impactos paisajísticos del proyecto en el entorno donde se desarrolla, establece un plan adecuado para la recuperación de los impactos generados con el fin de integrar el proyecto al entorno ambiental y diseña un mecanismo óptimo para el proceso de evaluación de impacto ambiental en los proyectos, basado en la valoración paisajística de los impactos.

Por otra parte, Niño (2018) desarrolló un modelo de zonificación para inducir restricciones a la minería en la región de La Macarena, departamento del Meta (Colombia), el cual integró la EAE al modelado de múltiples criterios, particularmente Electre Tri. El área de estudio se clasificó en cuatro niveles de restricción, de acuerdo a la jerarquía de mitigación de impactos: áreas excluidas y áreas de restricciones mayores, medias y menores; donde se podrían plantear intervenciones para evitar, minimizar, reparar, restaurar y compensar los efectos negativos ante un eventual desarrollo minero. Concluyó que el modelo de zonificación corresponde a una herramienta analítica, dinámica y multivariante de ordenamiento minero, donde el acopio, cruce y análisis de potencialidades, restricciones y condicionantes para el desarrollo de la minería en escenarios complejos, son de carácter prioritario.

\subsection{Debilidades en la apliación de la EAE}

El proceso de EAE no es perfecto, pero se encuentra en desarrollo y evolución, existen diferentes dificultades que impiden su puesta en práctica de manera efectiva. Algunos aspectos que influyen en su limitación son:

- Pocas entidades tienen experiencia en la aplicación de la EAE.

- Proceso novedoso, que su uso ha sido muy limitado sobre todo en el continente africano, donde ha sido motivado fundamentalmente por agencias de financiamiento internacional.

- Los resultados de la EAE debe ser integrados en el proceso de toma de decisión político, la cual puede tener una agenda distinta.

- La EAE en el ámbito internacional no se aplica a los niveles más altos de la toma de decisiones, que corresponden a políticas, planes y programas. En ese sentido, no está dirigida hacia la evaluación de alternativas en esos niveles ni hacia la evaluación de proyectos, en estos por su parte se aplica solamente la evaluación de impacto ambiental (EIA).

- Dentro del desarrollo de la EAE generalmente no se consideran los impactos acumulativos.

- La EAE no posee aún una metodología universal. 


\section{CONCLUSIONES}

La revisión bibliográfica realizada y la experiencia del autor en la materia, permite formular algunas afirmaciones de forma bastante categórica: Conceptualmente la EAE precede a la EIA y se constata un aumento progresivo del número y diversidad de enfoques procedimentales $\mathrm{y}$ metodológicos, desde la promulgación de la NEPA, para adaptarse a la enorme variedad que muestran los instrumentos de planificación que preceden a los proyectos.

El panorama más amplio, en cuanto avances en aplicación y metodología de EAE y en obligatoriedad, se encuentra en el continente europeo, donde mediante directivas de la comunidad Europea han logrado la estandarización metodológica y de exigencia para la contratación en proyectos de gran envergadura.

Para el continente Africano y especialmente para Angola, la EAE es un tema novedoso susceptible de avances en el desarrollo de herramientas de evaluación y control, los principales obstáculos y limitaciones para aplicar EAE, se relacionan con: 1) ausencias de normas relativas a la $\mathrm{EAE}$; 2) limitaciones de la capacidad de las instituciones y 3) dificultades relacionadas a la disponibilidad de información.

Teniendo en cuenta las caractetísticas del continente africano y particularme Angola, se propone, la metodología propuesta por el SEAN, para la aplicación a la actividad minera debido a las ventajas de dicha metodología, además que la misma fue creada para ser aplicada en los países de este continente.

\section{REFERENCIAS BIBLIOGRÁFICAS}

ADB (Asian Development Bank). (2014). Energy in Asia and the Pacific. Tomado de: http://www.adb.org/sectors/energy/main. Consultado en: abril, 2019.

Ahumada, C., Pelayo, T. \& Arano, C. (2012). Sustentabilidad ambiental, del concepto a la práctica. Una oportunidad para la implementación de la evaluación ambiental estratégica en México. Gestión y Política Pública. 21(2). pp. 291-332.

Banco Mundial. (2005). Integrating Environmental Considerations in Policy Formulation.

Lessons from Policy-Based SEA, Informe Número 32783.
http://www.wds.worldbank.org/external/default/WDSContentServer/WDSP/IB/2006/3/13/00016 0016 20060313104043/Rendered/PDF/327830white0co1vironmental01public1.pdf

Bayo, M. (2009) Experiencia Aplicada de EAE en América Latina y el Caribe EAE del Plan Energético Nacional de República Dominicana. Curso de EAE de Políticas, Planes y Programas. TAU Consultora Ambiental. San Carlos, Costa Rica.

Bina, O. (2008). Strategic Environmental Assessment. En Jordan, Andrew y Andrea Lenschow (Eds.) Innovation in Environmental Policy. Integrating environment for sustainability. CheltenhamUK. Edward Edgar Publishing Ltd. 
Bina, O., Xu, H., Brown, A., \& Partidario, M. (2011). Review of practice and prospects for SEA in China. Environmental Impact Assessment Review. 31, pp. 515-520.

Brown, A. \&Therivel, R. (2000). Principles to guide strategic environmental assessment methodology, in Impact Assessment and Project Appraisal. 18(3), pp. 183-190.

Calzada, J. (2014). La evaluación estratégica del impacto paisajístico en los pasivos mineros y su rentabilidad ambiental (tesis doctoral). Escuela Técnica Superior de Ingeniería Industrial. Universidad de la Rioja. 302 pp.

CEAA (Canadian Environmental Assessment Agency). (2014). Departmental Performance Report. Disponible en: http://www.ceaa-acee.gc.ca/default.asp?lang=En\&n=2ED690711\&offset=1\&toc=show\#s1.2. . Consultado: febrero 2019.

CED (Centro de Estudios del Desarrollo). (2010). Consultoría "marco conceptual para la implementación de la evaluación ambiental estratégica (EAE) en Chile". Capitulo II pág. 22, consultado el 15 de marzo de 2019 de http://portal.mma.gob.cl/evaluacion-ambiental-estrategica/

Charry, G. (2010) Propuesta metodológica para la evaluación ambiental estratégica del plan nacional de exploración y explotación de hidrocarburos en Colombia, Universidad Nacional de Colombia, Sede Bogotá, Instituto de Estudios Ambientales Facultad de Ciencias Económicas, 155 pp.

Clark, B. (1997) Alcances y objetivos de la Evaluación Ambiental Estratégica (EAE), Estudios Públicos 65, pp. 1-22.

Dalal-Clayton, B. \& Sadler, B. (2005) Strategic Environmental Assessment. A sourcebook and reference Guide to International Experience. Earthscan, USA.

Dalal-Clayton, D. (2004). The MDgs and sustainable Development: the need for a strategic Approach, en satterthwaite D. (ed) the Millennium Development goals and local processes: Hitting the target or Missing the point? international institute for environment and Development, london. pp 73-90.

Dalkmann, H., Herrera, R. \& Bongardt, D. (2004), Analytical Strategic Environmental Assessment (ANSEA) Developing a New Approach to SEA, Environmental Impact Assessment Review. 24(4), pp. 385-402.

Enriquez, S. \& Sanchez-Triana, E. (2005). Using Strategic Environmental Assessments for Environmental Mainstreaming in the Water and Sanitation Sector: The Cases of Argentina and Colombia. Latin America and Caribbean Region/ Environmentally and Socially Sustainable Development Department (LCSES). Development Working Paper 26. World Bank.

EPA (Environmental Protection Agency). (2012). Strategic Environmental Assessment. A review of its Effectiveness in Ireland. Ireland's Environmental Protection Agency. Ireland.

Espinoza, G. (2011). Experiencias de EAE en America Latina. Centro de Estudios del Desarrollo (CED). Santiago de Chile, Chile. Disponible en: http://www.ced.cl/ced/wpcontent/uploads/2011/10/eae-en-lac.pdf. Consultado: febrero 2019.

European Comission Environment. (2014). (Website) Tomado de: http://ec.europa.eu/environment/eia/home. Consultado: febrero 2019 
Facetti, J. (2003). Evaluación ambiental estratégica para elaboracion de politicas, planes y programas, Comparación de metodologias. Manual Técnico Para la Gestión del Saneamiento Ambiental. Ed. I, v. II, Asunción, Argentina.

Fevero, G. \& Katz, R. (1996). La Evaluación Ambiental Estratégica (EAE) y su aplicación a Políticas, Programas y Planes. Revista Estudios Públicos. 64, Universidad Católica de Chile.

Gómez, O. (2007). Evaluacion ambiental estratégica un instrumento para integrar el medio ambiente en la elaboración de planes y programas. Ediciones mundi-prensa. 366 pp.

lavH (Instituto de Investigaciones Alexander Von Humboldt). 2007. Plan Estratégico de Biodiversidad para el Desarrollo: El manejo sostenible de ecosistemas como aporte al bienestar humano. Colombia.

IDB (Inter-American Development Bank). (2004). Strategic Environmental Assessment for the CCLIP. IDB, Washington, D.C.

Jiliberto, R. \& Bonilla, M. (2009). Guía de Evaluación Ambiental Estratégica. Comisión Económica para América Latina y el Caribe (CEPAL). Naciones Unidas. Santiago de Chile.

Jiricka, A. (2008). SEA in local land use planning- first experience in the Alpine States. Environ Impact Asses Rev; 28, pp. 328-337.

Kulsum, A. \& Sánchez-Triana, E. (2009). Evaluación ambiental estratégica para la formulación de políticas. Un instrumento para la buena gobernabilidad. Banco Mundial en coedición con Mayol Ediciones S.A. 248pp.

Lobos, V. \& Partidario, M. (2014). Theory versus practice in Strategic Environmental Assessment (SEA). Environmental Impact Assessment Review 48, pp. 34-46. http://dx.doi.org/10.1016/j.eiar.2014.04.004

Marrero, B., Martínez, H., \& Quintana, O. (2013). La evaluación ambiental estratégica como herramienta para el ordenamiento ambiental y el desarrollo sostenible en Cuba. Propuesta metodológica. Revista Cubana de Geografía. 1, pp. 89-103.

Menéndez, N. (2017). Evaluación ambiental estratégica (EAE): Proceso de decisión ambiental y sostenible para Ecuador. Revista San Gregorio, 19, julio-diciembre, pp. 124-135.

Mercier, J. \& Ahmed, K. (2004). EIA and SEA at the World Bank. Paper presented to the proceedings of the 8th forum of the Intergovernmental Policy Forum on Environmental Assessment, Vancouver, Canada.

MMA - Ministério do Meio Ambiente. (2002). Avaliação Ambiental Estratégica. Secretaria de Qualidade nos Assentamentos Humanos. Brasília: MMA/SQA, pp. 92. Disponível en http://www.mma.gov.br/estruturas/sqa_pnla/_arquivos/aae.pdf. Consultado en: enero 2019.

Molina, U. \& Escalona, U. (2012). Evaluación ambiental estratégica: Instrumento para la planificación territorial urbana. Revista URBANO. 25, pp. 17-30.

MRC (Mekong River Comission). (2010). Strategic Environmental Assessment of Hydropower on the Mekong Mainstream. Final Report. International Centre for Environmental Management Disponible en: http://www.mrcmekong.org/assets/Publications/Reports/RSAT-Revision-3-forprinting consultado: Enero 2019. 
Nigel, R., Michelle, A., Paul, L., Stuart, H. \& Keith, W. (2000) Development of strategic environmental assessment in South Africa. Impact Assessment and Project Appraisal. 18(3), pp. 217-223. DOI: 10.3152/147154600781767394

Niño, L. (2018). Zonificación minera basada en la integración de la evaluación ambiental estratégica y modelado con múltiples criterios en la región de La Macarena, departamento del Meta, Orinoquía Colombiana. BioLlania Edición Esp. 15, pp. 634-666.

Noble, B. (2000). Strategic environmental assessment. What is it \& what makes it strategic. Journal of Environmental Assessment Policy and Management. 2(2), pp. 203-224.

Oberling, F. (2013). Desenvolvimento e aplicação de metodologia de Avaliação Ambiental Estratégica ao Planejamento da expansão do plantio de Florestas e de biocombustíveis líquidos. Rio de Janeiro. COPPE/UFRJ, 2013.

OCDE (Organización para la Cooperación y el Desarrollo Económicos). (2006). Applying Strategic Environmental Assessment (SEA): Good Practice Guidance for Development Cooperation. Development Assistance Committee Guidelines and Reference Series. París: OECD Publishing.

OECD-DAC 2006. Guía de buenas prácticas para la aplicación de evaluación ambiental estratégica (EAE) en la cooperación para el desarrollo. París: OCDE http://www.seataskteam.net/guidance.php.

Partidario, M. (1999). Strategic Environmental Assessment - Principles and Potential, en PettS, J. (ed.), Handbook of Environmental Impact Assessment, vol. 1, Oxford, Blackwell.

Partidario, M. (2007). Scales and associated data what is enough for SEA needs? Environ Impact Assess Rev. 27, pp. 460-78.

Partidario, M. (2011). Strategic Environmental Assessment (SEA): Current practices, Future Demands and Capacity-building needs. IAIA training course manual. (www.iaia.org).

Partidario, M. (2012). Strategic Envrionmental Assessment Better Practice Guide methodological guidance for strategic thinking in SEA. Lisbon, Portugal. http://ec.europa.eu/environment/eia/pdf/2012\%20SEA Guidance Portugal.pdf

Partidario. M. \& R. Clark. (2000). Perspectives on strategic environmental assessment. Lewis Publishers. Boca Raton. FI. USA.

Pérez, G. (2017) La Evaluación Ambiental Estratégica como instrumento de protección del medio en el Estado Autonómico. La Comunitat Valenciana como caso de estudio. Revista de Evaluación de Programas y Políticas Públicas. 8, pp. 31-53. http://dx.doi.org/10.5944/reppp.8.2017.16319

Pineda, J. (2006) Aplicación de la metodología de evaluación ambiental estratégica (EAE) al plan de exploración y explotación de hidrocaburos costa afuera en el Mar Caribe. (Tesis de grado) Universidad de la Salle, Facultad de Ingenieria Ambiental y Sanitaria, Bogota, Colombia. 142 pp.

Pirillo, E., D. Murguía., O, Cura. \& P, Ramati. (2012). Necesidad de una EAE de la minería metalífera en Argentina. Anales del $32 \circ$ Congreso Anual de la International Association for Impact Assessment. 27 Mayo - 10 Julio. Porto -Portugal. 7 pp. 
Rajvanshi, A. \& Mathur, V. (2005). Integrating Biodiversity into Strategic Environmental Assessment. Case Studies from India. Chandrabani: Wildlife Institute of India.

Retief, F., Jones, C., \& Jay, S. (2008). The emperor's new clothes. Reflections on strategic environmental assessment (SEA) practice in South Africa. Environmental Impact Assessment Review 28, pp. 504-514.

Sadler, B. \& Verhee, M. (1996). Strategic Environmental Assessment: Status, Challenges and Future Directions, The Netherlands, Ministry of Housing, Spatial Planning and the Envi-ronment, Report 53.

Sadler, B. (2008). International Trends and Developments in SEA Process and Practice. Center of Strategic Environmental Assessment for China, the Chinese University of Hong Kong, Hong Kong Institute of Environmental Impact Assessment, Research Centre for Strategic Environmental Assessment, Nankai University. Beijing. China.

Sanz, L. \& Sánchez, A. (1995). Medio ambiente y sociedad. De la metáfora organicista a la preservación ecológica. Granada: Comares.

Sinclair, A., John, S., \& Spaling, L. (2009). Community based approaches to strategic environmental assessment: Lessons from Costa Rica. Environmental Impact Assessment Review. 29 (3), pp. 147-156.

Tao, T., Tan, Z., \& He, X. (2007). Integrating environment into land-use planning through strategic environmental assessment in China: Towards legal frameworks and operational procedures. Environmental Impact Assessment Review 27, pp. 243-265.

Thérivel, R., Wilson, E., Thompson, S., Heaney, D. \& Pritchard, D. (1992). Strategic Environmental Assessment, Londres, Earthscan Publications, 1992. 181 pp.

Toro, J. (2009). Análisis constructivo del proceso de Evaluación de Impacto Ambiental en Colombia. Propuesta de Mejora (Tesis Doctoral). Universidad De Granada, España.

UICN/ORMAU. (2007). Lineamientos para la aplicación de la Evaluación Ambiental Estratégica en Centroamérica. UICN, San José, Costa Rica. 40 pp.

USHUD (United States Housingand Urban Development). (1981). Dept, Areawide Environmental Impact Assessment: a Guidebook, Washington D.C., Department of Housing and Urban Development.

Wood, C. (1992) Strategic environmental assessment in Australia and New Zealand, Project Appraisal, 7(3), pp. 143-149, DOI: 10.1080/02688867.1992.9726855

Wu, J., Chang, I., Bina, O., Lam, K., \& Xu, H. (2011). Strategic environmental assessment implementation in China - Five-year review and prospects. Environmental Impact Assessment Review. 31, pp. 77-84. 